Article

\title{
Agro-Morphological, Biochemical and Antioxidant Characterization of a Tunisian Chili Pepper Germplasm Collection
}

\author{
Karima Lahbib ${ }^{1, *(\mathbb{D} \text {, Samia Dabbou }}{ }^{2}$ D, Fethi Bnejdi ${ }^{1}$, Gaetano Pandino ${ }^{3, *(\mathbb{D} \text {, Sara Lombardo }}{ }^{3}$, \\ Mohamed El Gazzah ${ }^{1}$ and Safia El Bok ${ }^{1}$
}

1 Laboratory of Biodiversity, Biotechnology and Climate Changes, Faculty of Sciences of Tunis, University of Tunis El Manar, El Manar II, Tunis 2092, Tunisia; fethi.benejdi@issacm.u-sousse.tn (F.B.); Mohamed.Elgazzah@fst.rnu.tn (M.E.G.); safia.elbok@fst.utm.tn (S.E.B.)

2 Unit of Bioactive and Natural Substances and Biotechnology UR17ES49, Faculty of Dentistry, University of Monastir, Avicenne Street, Monastir 5019, Tunisia; samia.dabbou@fmdm.u-monastir.tn

3 Department of Agriculture, Food and Environment, University of Catania, Via Valdisavoia 5, 95123 Catania, Italy; sara.lombardo@unict.it

* Correspondence: karima.lahbib@fst.utm.tn (K.L.); g.pandino@unict.it (G.P.); Tel.: +216-73-46-08-32 (K.L.); Fax: +216-71-871 (K.L.)

check for updates

Citation: Lahbib, K.; Dabbou, S.; Bnejdi, F.; Pandino, G.; Lombardo, S.; El Gazzah, M.; El Bok, S. AgroMorphological, Biochemical and Antioxidant Characterization of a Tunisian Chili Pepper Germplasm Collection. Agriculture 2021, 11, 1236. https://doi.org/10.3390/ agriculture11121236

Academic Editor: Angeles Calatayud

Received: 6 October 2021

Accepted: 3 December 2021

Published: 8 December 2021

Publisher's Note: MDPI stays neutral with regard to jurisdictional claims in published maps and institutional affiliations.

Copyright: (c) 2021 by the authors. Licensee MDPI, Basel, Switzerland. This article is an open access article distributed under the terms and conditions of the Creative Commons Attribution (CC BY) license (https:// creativecommons.org/licenses/by/ $4.0 /)$

\begin{abstract}
Pepper species have been described as being highly sensitive to climate change. Here, we discuss the variability of the agro-morphological and phytochemical responses of pepper cultivars in the context of ongoing climate changes during seven stages of maturity, including heat stress. The effects and interactions were calculated to determine the source of variation according to rising temperature. Capsaicin content (CAP), total phenolic (TPC) and flavonoid (TFC) levels and antioxidant activity (AA) were also determined at different harvest times (at 10, 25, 40, 55, 70, 85 and 100 days after anthesis, DAA). Agro-morphological data showed that the highest variation was recorded for fruit traits compared to flower and plant ones. In particular, calyx shape margin, calyx annular constriction, fruit shape at blossom end and fruit size had a significant impact on the morphologic diversity among accessions. Levels of bioactive compounds and antioxidant activity depended on the genotype and the harvest time. TPC and AA increased at 100 DAA, while TFC were highly detected at the early harvest. Principal component analysis (PCA) allowed us to separate three clusters with well-defined biochemical traits. In particular, regardless of harvest time, Baklouti Chébika, Baklouti Sbikha and Chaabani accessions presented higher levels of TPC, TFC and AA regardless of the considered harvest time. In conclusion, high genetic variability was noted within the analyzed pepper germplasm, thus suggesting the need for major consideration of both agro-morphological and biochemical traits for pepper breeding programs. The current research was conducted to facilitate better management under high-stress conditions due to global warming
\end{abstract}

Keywords: Capsicum annuum L.; accessions; agro-morphological parameters; polyphenolic compounds; antioxidant activity; genetic discrimination

\section{Introduction}

The atmospheric $\mathrm{CO}_{2}$ concentration has increased by approximately $50 \%$ since human activities have increased the abundance of heat-trapping gases in the atmosphere, exceeding 400 ppm [1]. The average surface air temperature has risen by 1.8 degrees Fahrenheit. The recent increase in the average temperature and the atmospheric $\mathrm{CO}_{2}$ concentration is already leading to global severe abiotic changes in climate. Therefore, plants such as pepper are expected to face abiotic stresses $[2,3]$.

The genus Capsicum comprises approximately 35 taxa, including only five cultivated species identified [4,5]. Pepper fruits are widely appreciated for their high nutritional value, as they are known as a source of alkaloids, holding various pharmacological properties, 
and for possessing polyphenols, especially phenolic acids and flavonoids, which reduce the risk of some cancer types and cardiovascular diseases [6-8]. Tunisia is the largest producer of chilies in the African continent [9], where the versatility of pedo-climatic conditions favors the cultivation of a large number of specifically adapted pepper accessions that differ in fruit characteristics. They have been partially investigated and data have revealed that some of these landraces contain remarkable amounts of capsaicin and antioxidant compounds, which are useful for the processing industries [10]. Specific consideration must be given to the genotype and harvest time, which are important factors in determining the antioxidant compounds present in fruits and vegetables [11-13]. With the target of enhancing these bioresources, the present study aimed to characterize eleven Tunisian chili pepper accessions for their agro-morphological and biochemical traits, evaluating the variation in antioxidant compounds in relation to harvest time.

\section{Materials and Methods}

\subsection{Plant Material}

Eleven autochthonous chili pepper accessions, derived from four sites located in the north and middle of Tunisia, were studied: 'BakloutiChébika' (BaklC), 'Beldi' (Bel), 'Chaabani' (Chba), 'SissebChébika' (SisC), 'Bkalti' (Bka), 'Knaiss' (Kna), 'BakloutiSbikha' (BaklS), 'SissebSbikha' (SisS), 'Fort Menzeltemim' (FkbM), 'Fort de korba' (FkbK) and 'Corne de Gazelle' (CGaz). Details on the native sites of such accessions were described in our previous paper [10]. The present trial was carried out at the experimental field of the University of Tunis, Faculty of Sciences, located in Northern Tunisia $\left(36^{\circ} 81^{\prime} 88^{\prime \prime} \mathrm{N}, 10^{\circ} 16^{\prime} 6^{\prime \prime} \mathrm{E}\right.$, $7.1 \mathrm{~m}$ a.s.1.), during the crop years 2016-2017. The experimental site is characterized by a Mediterranean maritime climate (humidity: $73 \%$; annual mean relative temperature: $20.1^{\circ} \mathrm{C}$; annual mean total rainfall: $415 \mathrm{~mm}$ ) and exhibits agro-climatic differences among the four sites of material collection (Figure 1).

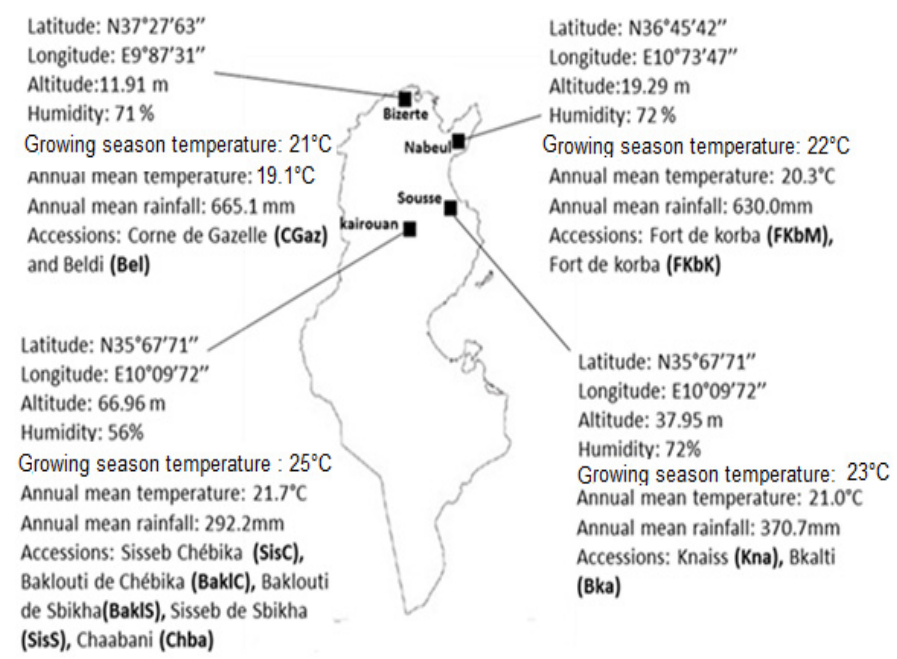

Figure 1. Geographical coordinates and annual climatic conditions in the four Tunisian sites where chili pepper accessions were collected.

The seeds were sown under greenhouse conditions and the seedlings were transplanted in the experimental field (humidity: 73\%; annual mean relative temperature: $20.1^{\circ} \mathrm{C}$; annual mean total rainfall: $415 \mathrm{~mm}$ ), including a complete randomized block with three replications, for a total of 55 plants per plot. Providing peppers with adequate water is essential, with 1 inch of water per week, and it is necessary to adjust the amount or frequency during hot, dry periods, after rainfall or if the soil is sandy and drains fast. Drip irrigation is ideal for growing peppers, because it is economical and less labor-intensive without wetting the foliage and possibly triggering diseases. In particular, organic fertilizers completely fermented and decomposed, such as compost and manure, were supplemented together with ammonium nitrate, at three time points: early season 
(2-30 days from planting; $2 \mathrm{~kg} \mathrm{ha}^{-1}$ ), main season (31-70 days from planting; $132 \mathrm{~kg} \mathrm{ha}^{-1}$ ) and end season (71-130 days from planting; $145 \mathrm{~kg} \mathrm{ha}^{-1}$ ). Pest control was undertaken according to the recommendations authorized by the Department of Agriculture, Ministry of Agriculture and Cooperatives. The applied rate of pesticide was determined according to the relative pest, followed by a survey of the disease infestation on all the plant parts and fruits.

\subsection{Agro-Morphological Characterization}

Agro-morphological data were collected from 10 plants and 20 fruits per replicate. Sixteen descriptive traits associated with chili pepper plant, flower and fruit were evaluated on the basis of the Capsicum descriptors developed by the International Plant Genetic Resources Institute [14]. The plant traits included: growth habit (GH), leaf density (LD), leaf pubescence (LP), nodal anthocyanin (NA). The flower traits consisted of corolla color (CC), flower position (FP), calyx shape margin (CSM), calyx annular constriction (CAC). Fruit characters included: fruit color in mature stage (FCM), fruit color in immature stage (FCI), fruit position (FrP), fruit shape at pedicel attachment (FSP), fruit shape at blossom end (FSB), fruit cross-sectional corrugation (FCC). Biometric traits were fruit fresh weight (FFW, expressed as g) measured with an FA-G series electromagnetic balance, fruit length ( $\mathrm{FL}$, expressed as $\mathrm{cm}$ ) and fruit diameter ( $\mathrm{FD}$, expressed as $\mathrm{cm}$ ) determined with a digital caliper. The harvest was performed from mid-July to mid-September and was recorded as the number of fruits per plant $(\mathrm{NF} / \mathrm{Pl})$.

\subsection{Biochemical Analysis}

Pepper fruits were harvested every 15 days (at 10, 25, 40, 55, 70, 85 and 100 days after anthesis (DAA)) and the daily mean temperatures were calculated from measurements taken before each harvest (Table 1). Physiological maturity has been defined by Watada et al. (1984) as "the stage of development when a plant or plant part will continue ontogeny even if detached" while horticultural maturity has been defined as "the stage of development when a plant or plant part possesses the prerequisites for utilization by consumers for a particular purpose". In this study, we propose an integrative approach using days after anthesis and post-harvest studies to define physiological and horticultural maturity, as used by Neves et al. [15].

Table 1. Air temperature at harvest, fruit maturity and color of the eleven Tunisian chili pepper accessions at each harvest time.

\begin{tabular}{cccc}
\hline $\begin{array}{c}\text { Harvest Time } \\
\text { (Days after Anthesis, DAA) }\end{array}$ & $\begin{array}{c}\text { Air Temperature at } \\
\text { Harvest }\left({ }^{\circ} \mathbf{C}\right)\end{array}$ & $\begin{array}{c}\text { Horticultural } \\
\text { Maturity }\end{array}$ & Fruit Color \\
\hline 10 & 25.2 & Unripe & fully green \\
25 & 28.8 & Half ripe & fully green \\
40 & 31.4 & Ripe & fully green \\
55 & 34.1 & Ripe & orange \\
70 & 36.2 & Ripe & red \\
85 & 37.5 & Over ripe & red \\
100 & 38.4 & Over ripe & dark red \\
\hline
\end{tabular}

Samples were collected (Table 1) from all cultivars at different harvest times and horticultural maturity: 10 DAA (unripe, fully green), 25 DAA (half ripe, fully green), 40 DAA (ripe, fully green), 55 DAA (ripe, orange), 70 DAA (ripe, red), 85 DAA (over ripe, red) and 100 DAA (over ripe, dark red) and were dried at $30{ }^{\circ} \mathrm{C}$ until a constant weight was reached and then were subjected to biochemical analyses. 


\subsubsection{Nutraceutical Determination}

The capsaicin (CAP) content was evaluated in the chili pepper fruits following the procedure reported in previous works [10,16]. Briefly, two grams were crushed in $10 \mathrm{~mL}$ of acetone using pestle and mortar. The obtained solution was then centrifuged, and the supernatants were recuperated. The supernatants were evaporated to dryness and re-suspended in $0.4 \mathrm{~mL}$ of $\mathrm{NaOH}$ and $3 \mathrm{~mL}$ of $3 \%$ phosphomolybdic acid. After shaking, the resulting solution was allowed to stand for $1 \mathrm{~h}$ at room temperature, filtered and centrifuged. A UV-Vis spectrophotometer (BioSpectrometer ${ }^{\circledR}$ Series, Hamburg, Germany) was used to measure the absorbance of the clear blue solution obtained at $650 \mathrm{~nm}$. The CAP amount was expressed as mg of CAP equivalents $\mathrm{g}^{-1}$ of dry weight (DW).

Extracts for the determination of total phenols (TPC), total flavonoids (TFC) and antioxidant activity analysis were prepared according to Lahbib et al. [10]. The TPC was determined by the method of Folin-Ciocalteu [17] and expressed as mg gallic acid equivalents (GAE) $\mathrm{g}^{-1}$ DW. For the TFC, the method of Um and Kim [18] was adopted, reading the absorbance at $430 \mathrm{~nm}$ with a UV-Vis spectrophotometer (BioSpectrometer ${ }^{\circledR}$ Series, Hamburg, Germany). TFC content was expressed as mg naringin equivalent (NAE) $\mathrm{g}^{-1}$ DW.

\subsubsection{Antioxidant Activity}

The DPPH (1,1-diphenyl-2-picrylhydrazyl) assay was used to measure the antioxidant activity [19]. The results were expressed as DPPH percentage inhibition, as reported previously [10].

The ferric reducing antioxidant power (FRAP) was evaluated with the UV-Vis spectrophotometer according to the procedure of Benzie and Strain [20]. The antioxidant activity was expressed as Trolox (6-hydroxy-2,5,7,8-tetramethylchroman-2-carboxylic acid) equivalents in $\mathrm{mmol} \mathrm{g}^{-1} \mathrm{DW}$.

\subsection{Statistical Analysis}

All traits were subjected to univariate and multivariate analysis. Biometric data were examined by analysis of variance (ANOVA) using a 95\% confidence interval, followed by means comparison with Duncan's test and correlation analysis to determine associations among the studied traits. Descriptive characters were expressed as relative frequencies for all and each accession. Principal component analysis (PCA) and hierarchical cluster analysis (HCA) were used to obtain a general overview of the variation among the studied chili pepper accessions. All the statistical analyses were performed by using XLSTAT for Windows (Addinsoft, New York, NY, USA).

\section{Results and Discussion}

\subsection{Agro-Morphological Characterization}

Large phenotypic variation was observed, especially for nodal anthocyanin (NA), calyx shape margin (SCM), fruit shape at pedicel attachment (FSP), fruit shape at the blossom end (FSB) and fruit cross-sectional corrugation (FCC) (Table 2). The fruit shape at blossom end was mainly blunt (FSBb), reaching $58.7 \%$ relative frequency, while the fruit shape at pedicel attachment was more variable among the studied chili pepper accessions, ranging from acute $(38.9 \%)$ or obtuse (FSPo) $(34.7 \%)$ to truncate (FSPt) $(26.4 \%)$. All fruits were slightly (FCCs) to intermediately (FCCi) corrugated (Table 2).

Bel, Kna and Bka accessions exhibited the highest NF/Pl (73.3, 44.9 and 44.9, respectively). Contrastingly, it was observed that both the FFW and FL/FD ratio were lower in Bel $(8.6 \mathrm{~g}, 3.0)$, Bka $(9.7 \mathrm{~g}, 2.4)$ and Kna $(9.8 \mathrm{~g}, 2.8)$ than in FKbK $(12.8 \mathrm{~g}, 8.6)$ and FKbM (12.1 g, 8.5) (Table 3). 
Table 2. Frequency distribution of the 16 descriptive traits among the Tunisian chili pepper accessions under study.

\begin{tabular}{|c|c|c|c|c|c|c|c|}
\hline Trait (Acronym) & Description (Acronym) & \multicolumn{6}{|c|}{ Frequency Distribution } \\
\hline Growth habit $(\mathrm{GH})$ & $\begin{array}{c}\text { prostrate (GHp), compact (GHc), } \\
\operatorname{erect}(\mathrm{GHe})\end{array}$ & $\begin{array}{l}\text { GHp } \\
85.6\end{array}$ & $\begin{array}{c}\mathrm{GHc} \\
4.2\end{array}$ & $\begin{array}{l}\mathrm{GHe} \\
10.2\end{array}$ & & & \\
\hline Leaf density (LD) & $\begin{array}{l}\text { sparse (LDs), intermediate (LDi), } \\
\text { dense (LDd) }\end{array}$ & $\begin{array}{l}\text { LDs } \\
82.4\end{array}$ & $\begin{array}{l}\text { LDi } \\
15.0\end{array}$ & $\begin{array}{l}\text { LDd } \\
2.6\end{array}$ & & & \\
\hline Leaf pubescence (LP) & $\begin{array}{c}\text { sparse (LPs), intermediate (LFi), } \\
\text { dense (LFd) }\end{array}$ & $\begin{array}{l}\text { LPs } \\
100\end{array}$ & $\begin{array}{c}\mathrm{LPi} \\
0\end{array}$ & $\begin{array}{c}\mathrm{LPd} \\
0\end{array}$ & & & \\
\hline Nodal anthocyanin (NA) & $\begin{array}{c}\text { green (NAg), light purple (NAl), } \\
\text { purple (NAp), dark purple (NAd) }\end{array}$ & $\begin{array}{l}\text { NAg } \\
17.0\end{array}$ & $\begin{array}{l}\text { NAlp } \\
21.0\end{array}$ & $\begin{array}{l}\text { NAp } \\
30.0\end{array}$ & $\begin{array}{l}\text { NAdp } \\
32.0\end{array}$ & & \\
\hline Corolla color (CC) & $\begin{array}{l}\text { white }(\mathrm{CCw}) \text {, green white }(\mathrm{CCgw}) \text {, } \\
(\mathrm{CCl}) \text {, blue }(\mathrm{CCb}) \text {, violet }(\mathrm{CCv})\end{array}$ & $\begin{array}{c}\mathrm{CCw} \\
100\end{array}$ & $\begin{array}{c}\mathrm{CCg} \\
0\end{array}$ & $\begin{array}{c}\mathrm{CCl} \\
0\end{array}$ & $\begin{array}{c}\mathrm{CCb} \\
0\end{array}$ & $\begin{array}{c}\mathrm{CCv} \\
0\end{array}$ & \\
\hline Flower position (FP) & $\begin{array}{c}\text { pendant (FPp), intermediate (FPi), } \\
\text { shoot (FPs) }\end{array}$ & $\begin{array}{l}\text { FPe } \\
76.0\end{array}$ & $\begin{array}{c}\mathrm{FPi} \\
15.0\end{array}$ & $\begin{array}{c}\text { FPs } \\
9.0\end{array}$ & & & \\
\hline Filament color (FC) & blue $(\mathrm{FCb})$, white $(\mathrm{FCw})$ & $\begin{array}{c}\mathrm{FCw} \\
0\end{array}$ & $\begin{array}{c}\mathrm{FCb} \\
100\end{array}$ & & & & \\
\hline Calyx shape margin (CSM) & $\begin{array}{l}\text { smooth (CSMs), intermediate } \\
\text { (CSMi), dentate (CSMd) }\end{array}$ & $\begin{array}{l}\text { CSMs } \\
20.9\end{array}$ & $\begin{array}{c}\text { CSMi } \\
56.9\end{array}$ & $\begin{array}{c}\text { CSMd } \\
22.2\end{array}$ & & & \\
\hline Calyx annular constriction (CAC) & absent (CACa), present (CACp) & $\begin{array}{l}\text { CACa } \\
30.5\end{array}$ & $\begin{array}{c}\text { CACp } \\
69.5\end{array}$ & & & & \\
\hline Fruit position (FrP) & $\begin{array}{l}\text { declining } \mathrm{n}(\mathrm{FrPd}) \text {, intermediate } \\
(\mathrm{FrPi}) \text {, erect }(\mathrm{FrPe})\end{array}$ & $\begin{array}{c}\mathrm{FrPd} \\
53.1\end{array}$ & $\begin{array}{l}\text { FrPi } \\
40.1\end{array}$ & $\begin{array}{c}\text { FrPe } \\
6.8\end{array}$ & & & \\
\hline Fruit color in immature stage (FCI) & $\begin{array}{c}\text { green }(\mathrm{FCIg}), \text { yellow (FCIy), orange } \\
\text { (FCIo), red (FCIr), purple (FCIp), } \\
\text { brown (FCIb) }\end{array}$ & $\begin{array}{l}\text { FCIg } \\
100\end{array}$ & $\begin{array}{l}\text { FCIy } \\
0\end{array}$ & $\begin{array}{c}\text { FCIo } \\
0\end{array}$ & $\begin{array}{c}\text { FCIr } \\
0\end{array}$ & $\begin{array}{l}\text { FCIp } \\
0\end{array}$ & $\begin{array}{c}\mathrm{FCIb} \\
0\end{array}$ \\
\hline Fruit color in mature stage (FCM) & $\begin{array}{c}\text { green }(\mathrm{FCMg}) \text {, yellow }(\mathrm{FCMy}) \\
\text { orange }(\mathrm{FCM}) \text {, red }(\mathrm{FCMr}) \text {, purple } \\
(\mathrm{FCMp}), \text { brown }(\mathrm{FCMb})\end{array}$ & $\begin{array}{c}\mathrm{FCMg} \\
0\end{array}$ & $\begin{array}{c}\text { FCMy } \\
0\end{array}$ & $\begin{array}{l}\text { FCMo } \\
\quad 0\end{array}$ & $\begin{array}{c}\text { FCMr } \\
100\end{array}$ & $\begin{array}{l}\text { FCMp } \\
\quad 0\end{array}$ & $\begin{array}{l}\mathrm{FCMb} \\
0\end{array}$ \\
\hline $\begin{array}{l}\text { Fruit shape at pedicel attachment } \\
\qquad(\mathrm{FSP})\end{array}$ & $\begin{array}{c}\text { acute }((\mathrm{FSPa}), \text { obtuse }(\mathrm{FSPo}) \\
\text { truncate }(\mathrm{FSPt}), \text { cordate }(\mathrm{FSPc}) \\
\text { lobate }(\mathrm{FSPl})\end{array}$ & $\begin{array}{c}\text { FSPa } \\
38.9\end{array}$ & $\begin{array}{l}\text { FSPo } \\
34.7\end{array}$ & $\begin{array}{l}\mathrm{FSPt} \\
26.4\end{array}$ & $\begin{array}{c}\mathrm{FSPC} \\
0\end{array}$ & FSP1 & \\
\hline Fruit shape at blossom end (FSB) & $\begin{array}{l}\text { pointed (FSBp), blunt (FSPb), } \\
\text { sunken (FSPs) }\end{array}$ & $\begin{array}{l}\text { FSBp } \\
24.5\end{array}$ & $\begin{array}{c}\mathrm{FSBb} \\
58.7\end{array}$ & $\begin{array}{c}\text { FSBs } \\
16.8\end{array}$ & & & \\
\hline $\begin{array}{l}\text { Fruit cross sectional corrugation } \\
\text { (FCC) }\end{array}$ & $\begin{array}{c}\text { slight (FCCs), intermediate (FCCi), } \\
\text { high (FCCh) }\end{array}$ & $\begin{array}{c}\text { FCCs } \\
21.0\end{array}$ & $\begin{array}{c}\text { FCCi } \\
40.7\end{array}$ & $\begin{array}{l}\text { FCCh } \\
38.3\end{array}$ & & & \\
\hline
\end{tabular}

Table 3. Biometric traits of the eleven Tunisian chili pepper accessions under study.

\begin{tabular}{ccccc}
\hline Accession & Acronym & FL/FD & FFW (g) & NF/Pl \\
\hline Corne de Gazelle & CGaz & $4.2^{\mathrm{bc}}$ & $10.8^{\mathrm{bd}}$ & $35.0^{\mathrm{cd}}$ \\
BakloutiChébika & BaklC & $2.6^{\mathrm{de}}$ & $10.5^{\mathrm{cd}}$ & $38.0^{\mathrm{bc}}$ \\
SissebChébika & SisC & $5.0^{\mathrm{bc}}$ & $11.2^{\mathrm{ac}}$ & $32.6^{\mathrm{cd}}$ \\
Beldi & Bel & $3.0^{\mathrm{d}}$ & $8.6^{\mathrm{e}}$ & $73.3^{\mathrm{a}}$ \\
SissebSbikha & SisS & $5.3^{\mathrm{b}}$ & $12.0^{\mathrm{ab}}$ & $27.1^{\mathrm{e}}$ \\
BakloutiSbikha & BaklS & $2.9^{\mathrm{d}}$ & $10.0^{\mathrm{d}}$ & $33.0^{\mathrm{cd}}$ \\
Knaiss & Kna & $2.8^{\mathrm{d}}$ & $9.8^{\mathrm{d}}$ & $44.9^{\mathrm{b}}$ \\
Bkalti & Bka & $2.4^{\mathrm{de}}$ & $9.7^{\mathrm{d}}$ & $44.9^{\mathrm{b}}$ \\
Chaabani & Chba & $2.0^{\mathrm{e}}$ & $11.6^{\mathrm{ac}}$ & $33.1^{\mathrm{cd}}$ \\
Fort de korba & FKbK & $8.6^{\mathrm{a}}$ & $12.8^{\mathrm{a}}$ & $15.8^{\mathrm{f}}$ \\
Fort Menzeltemim & FKbM & $8.5^{\mathrm{a}}$ & $12.1^{\mathrm{a}}$ & $18.8^{\mathrm{f}}$ \\
\hline
\end{tabular}

FL: fruit length; FD: fruit diameter; FFW: fruit fresh weight; NF/Pl: number of fruits per plant. Different letters $\left({ }^{a-f}\right)$ within the same trait indicate significant differences among accessions $(p \leq 0.05)$. 
From PCA analysis (Table 4), the agro-morphologic characteristics of the fruit were the greatest contributors to genetic diversity and were associated with PC1. Similar trends have also been reported for other Capsicum species and closely related horticultural crops, including tomato $[5,21,22]$. Indeed, PC1 $(41.7 \%)$ was associated with calyx shape margin (CSM), calyx annular constriction (CAC), fruit shape at pedicel attachment (FSP), fruit shape at blossom end (FSB), nodal anthocyanin (NAg), fruit weight (FW) and number of fruits per plant (NF/Pl). PC2 (21.2\%) was correlated with flower position (FP), nodal anthocyanin (NA), growth habit (GHp), leaf density (LD) and fruit length/fruit diameter ratio (FL/FD) (Table 4).

Table 4. Variance estimates (eigenvalues) for the 25 evaluated traits in the eleven Tunisian chili pepper accessions.

\begin{tabular}{ccccc}
\hline Principal Component & Eigenvalue & Accumulated \% & Traits Correlated & Accessions Correlated \\
\hline PC1 & 15.8 & $\begin{array}{c}\text { CSM (calyx shape margin); CAC (calyx } \\
\text { annular constriction); FSP (fruit shape at } \\
\text { pedicel attachment); FSB (fruit shape at } \\
\text { blossom end); NAg (nodal anthocyanin); } \\
\text { FW (fruit weight); NF/Pl (number of } \\
\text { fruits per plant) }\end{array}$ & $\begin{array}{c}\text { FKK, FKbM, Kna, Bka } \\
\text { CGaz, SisS }\end{array}$ \\
\hline PC2 & & $\begin{array}{c}\text { FP (flower position); NA (nodal } \\
\text { anthocyanin); GHp (growth habit); LD } \\
\text { (leaf density); FL/FD (fruit length/fruit } \\
\text { diameter ratio) }\end{array}$ & BaklC, BaklS, Chba, \\
PC3 & 62.9 & $\begin{array}{c}\text { FrP (fruit position); FCC (fruit } \\
\text { cross-sectional corrugation) }\end{array}$ & Bel, SisC \\
\hline
\end{tabular}

A higher degree of dispersion was observed between and within the clusters formed (Figure 2). Such distribution was attributed to the influence of the different morphotypes of the different studied accessions, as observed by different authors $[4,23,24]$. From cluster analysis (Figure 2), no relation was found between the origin of the cultivars and the cluster pattern. This suggest that the Tunisian accessions could easily adapt in different cultivation areas as the climatic conditions of the experimental field were different from the original site. This indicates genetic variability within cultivars, and this would be helpful in selecting desirable chili pepper phenotypes.

\subsection{Biochemical Characterization \\ 3.2.1. Capsaicin Content}

The cultivars under study had CAP content varying from $0.20-0.26 \mathrm{mg} \mathrm{CAP} \mathrm{g}^{-1} \mathrm{DW}$ (at 10 DAA) to $0.32-0.63 \mathrm{mg} \mathrm{CAP} \mathrm{g}^{-1} \mathrm{DW}$ (at $\left.100 \mathrm{DAA}\right)$. The CAP content increased until 40 DAA, resulting in high values in Chba $\left(0.83 \mathrm{mg} \mathrm{CAP} \mathrm{g}^{-1} \mathrm{DW}\right)$, BaklC $\left(0.82 \mathrm{mg} \mathrm{CAP} \mathrm{g}^{-1}\right.$ DW) and BaklS (0.80 $\left.\mathrm{mg} \mathrm{CAP} \mathrm{g}^{-1} \mathrm{DW}\right)$. It significantly decreased from 40 to 85 DAA (Figure 3a), while at 100 DAA, the CAP content increased significantly. Fluctuation in CAP content, which occurred during ripening and was registered in the tested accessions, may have been due to variation in the levels of peroxidase (POD) enzymes, as a result of some environmental factors, such as temperature and soil moisture. Similar trends were observed by Anjum et al., Fayos et al., Barbero et al. and Ruiz-Lau et al. [25-28]. 
(a)

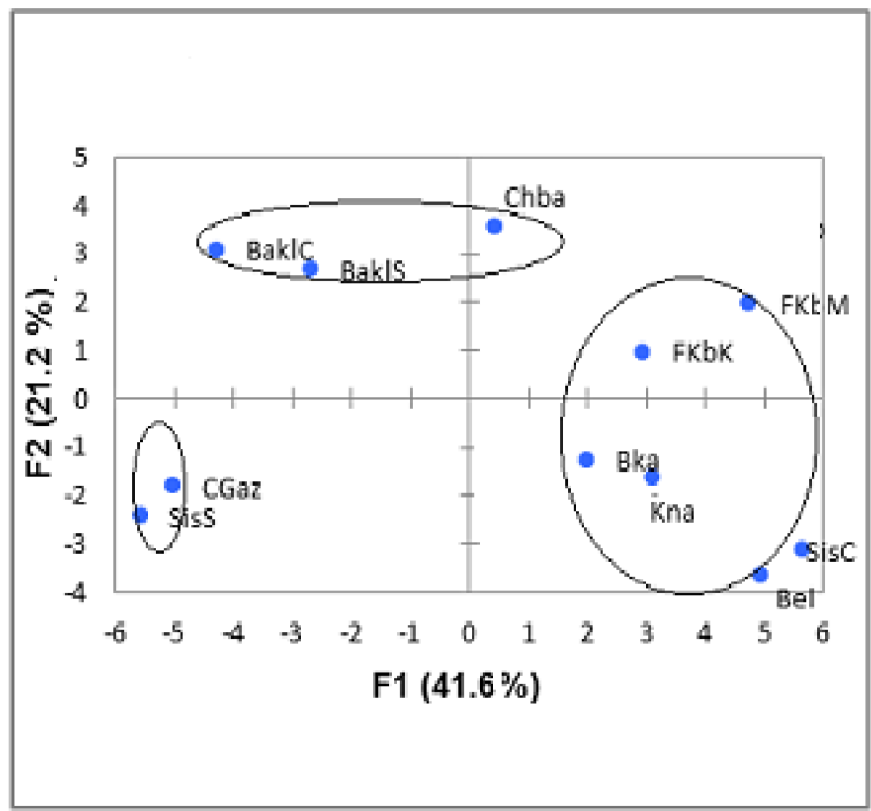

(b)

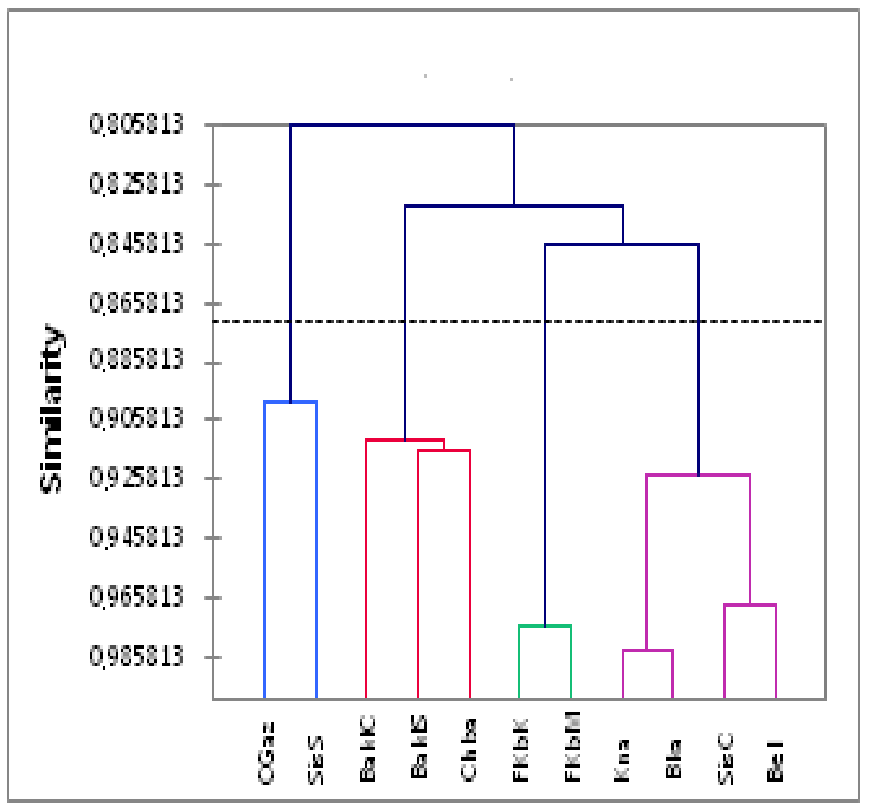

Figure 2. (a) Spatial distribution of the eleven Tunisian chili pepper accessions according to the first two PCA components based on the agro-morphological traits. (b) Dendrogram obtained from cluster analysis of the eleven Tunisian chili pepper accessions based on the analyzed quantitative and qualitative traits. 'Baklouti Chébika' (BaklC), 'Beldi' (Bel), 'Chaabani' (Chba), 'Sisseb Chébika' (SisC), 'Bkalti' (Bkaa), 'Knaiss' (Kna), 'Baklouti Sbikha' (BaklS), 'Sisseb Sbikha' (SisS),

'Fort Menzeltemim' (FkbM), 'Fort de korba' (FkbK), 'Corne de Gazelle' (CGaz).

\subsubsection{Total Phenol Content}

As observed in Figure 3b, TPC content varied with the harvest time. In relation to each harvest time, TPC oscillated from 2.01-4.10 $\mathrm{mg} \mathrm{GAE} \mathrm{g}^{-1} \mathrm{DW}$ (at 10 DAA) to 2.60-5.80 $\mathrm{mg} \mathrm{GAE} \mathrm{g}^{-1}$ DW (at 100 DAA). At 100 DAA, FKbM and BaklC had the highest levels (5.80 and $5.70 \mathrm{mg} \mathrm{GAE} \mathrm{g}^{-1} \mathrm{DW}$, respectively), while CGaz had the lowest (2.60 $\mathrm{mg} \mathrm{GAE} \mathrm{g}^{-1} \mathrm{DW}$ ) (Table 1). In particular, from 10 to $40 \mathrm{DAA}$, the TPC amount did not vary significantly (Figure $3 b$ ), probably due to the vigorous growth of the pepper plants at this time. By contrast, wide variation was observed from 55 to 100 DAA as a result of the availability of precursors of polyphenols at higher temperatures and the establishment of different antioxidant compounds with a different degree of antioxidant activity [29].

\subsubsection{Total Flavonoid Content}

The TFC significantly decreased from early to later harvest times (Table 4). In particular, a significant decrease in the TFC concentration was observed from $55\left(0.18-0.40 \mathrm{mg} \mathrm{NAE} \mathrm{g}^{-1}\right.$ DW) to 100 DAA (0.11-0.33 mg NAE g ${ }^{-1}$ DW). Both BaklC and BaklS showed the highest

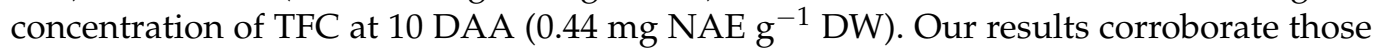
of previous studies by Ghasemnezhad et al. and Howard et al. [30,31]. Some environmental factors, such as light condition, water status and temperature, influence flavonoid amounts [32]. According to the present study, lower temperatures appeared favorable to flavonoid accumulation, as reported also by Pandino et al. [33] for globe artichoke. 
(a)

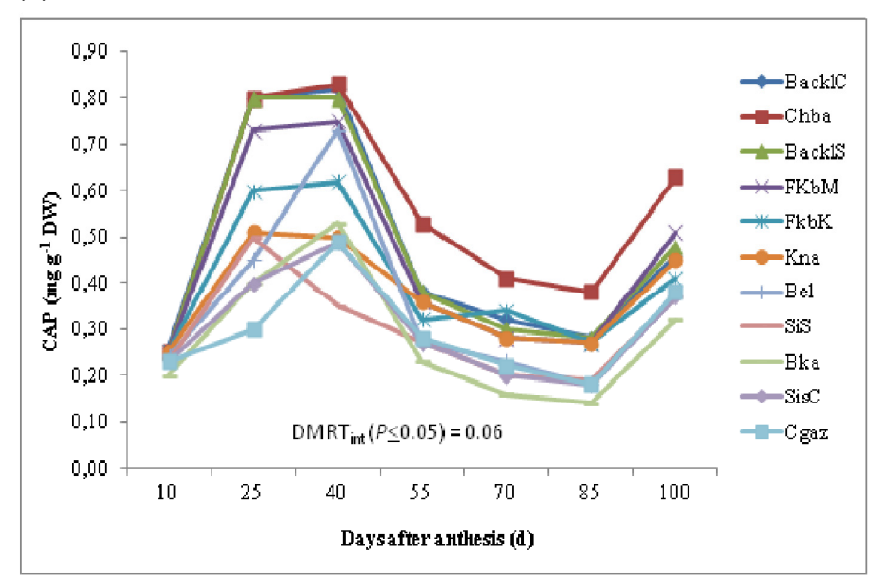

(b)

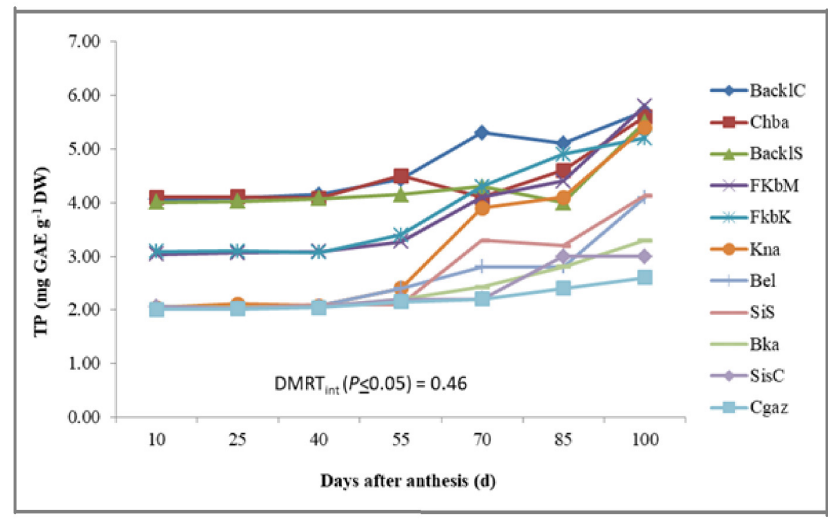

(d)

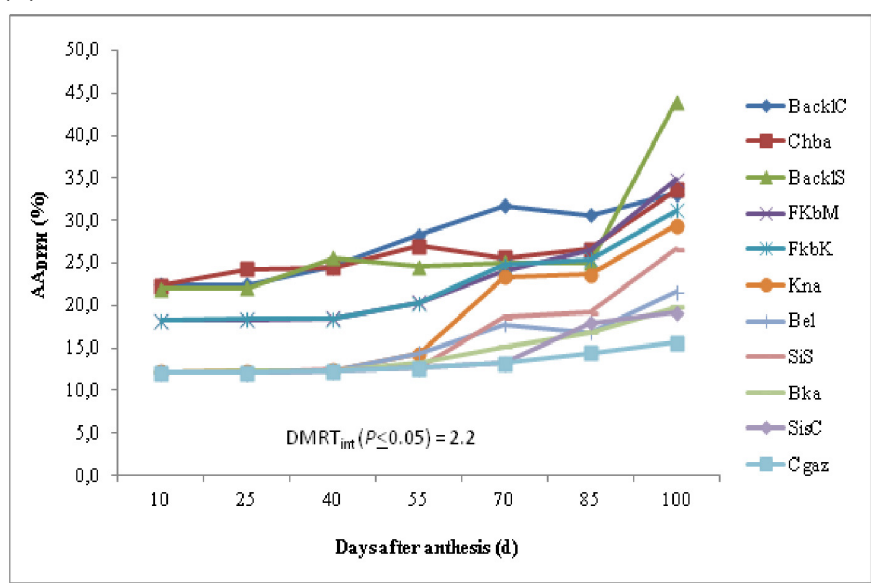

(c)

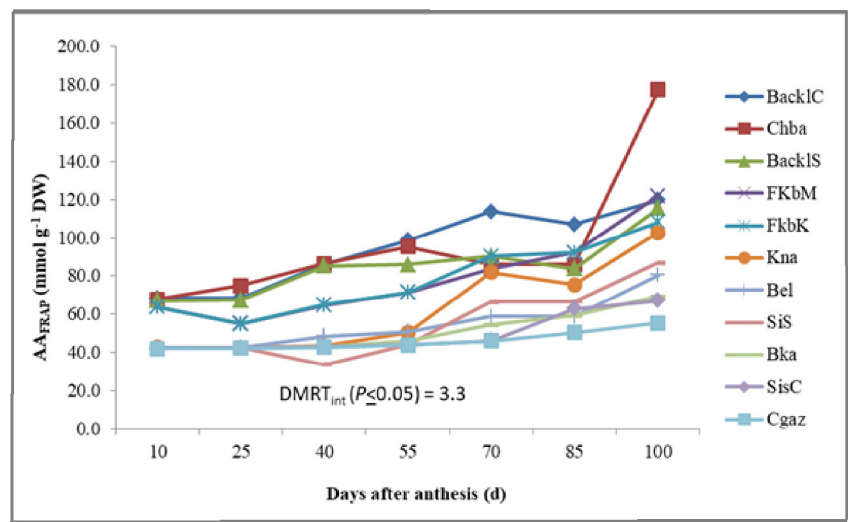

Figure 3. Variation in phytochemical compounds and antioxidant activity (by DPPH and FRAP assays) in the eleven Tunisian chili pepper accessions in relation to harvest time. CAP: capsaicin content (a). TPC: total phenol content (b). AADPPH and AAFRAP: antioxidant activity by DPPH (c) and FRAP (d) assays, respectively.

\subsubsection{Antioxidant Activity}

The antioxidant activity through DPPH ranged from 12.1 (SisS, CGaz and SisC) to 22.4\% (BaklC) at 10 DAA and from 15.6 (CGaz) to 44.0\% (BaklS) at 100 DAA (Table 4, Figure $3 \mathrm{c}$ ). Using the FRAP assay, the antioxidant activity ranged from 42.2 (SisS, GCaz and SisC) to $68.4 \mathrm{mmol} \mathrm{g}^{-1} \mathrm{DW}$ (BaklC) at $10 \mathrm{DAA}$ and from 55.6 (CGaz) to $121.8 \mathrm{mmol} \mathrm{g}^{-1} \mathrm{DW}$ (FkbM) at 100 DAA (Table 5, Figure 3d). Regardless of the assay adopted, the antioxidant activity significantly increased throughout the harvest time, as especially observed for FkbM, BaklC, Chba and BaklS (Figure 4c,d). This agrees with the previous findings of Howard et al. and Mitic et al. [31,34]. Thus, it is strongly recommended to consume hot pepper fruits at the latest stages of ripening.

\subsection{Analysis of Variance and Interaction}

All traits exhibited wide diversity among genotypes, even though the effect of the harvest stages had a greater influence on bioactive compound variation. Harvest time $(\mathrm{H})$ and accession $\times$ harvest time $(\mathrm{A} \times \mathrm{H})$ effects accounted for 16.4 to $79.9 \%$ and 0.6 to $1.6 \%$ of the total variance, respectively. Such results suggest the influence of the related air temperature values evidenced for the seven stages (Table 5). Indeed, the temperature, light intensity, plant nutrition and degree of maturation of the fruit are factors interfering in the metabolic activities during the development of the plant, being able to influence the concentration of phytochemical components [35]. Meckelmann et al. [36] concluded that 
the production of flavonoids depends strongly on the growing conditions, but the total polyphenols and antioxidant capacity (TEAC) do not vary to a large extent.

Table 5. Two-way ANOVA results for the analyzed biochemical traits of Tunisian chili pepper.

\begin{tabular}{|c|c|c|c|c|c|}
\hline Main Factor & $\begin{array}{c}\text { CAP } \\
\left(\mathrm{mg} \mathrm{g}^{-1} \mathrm{DW}\right)\end{array}$ & $\begin{array}{c}\text { TPC } \\
\left(\mathrm{mg} \mathrm{GAE} \mathrm{g}^{-1} \mathrm{DW}\right)\end{array}$ & $\begin{array}{c}\text { TFC } \\
\left(m g \text { NAE } g^{-1} \mathrm{DW}\right)\end{array}$ & $\begin{array}{l}\text { AADPPH } \\
(\%)\end{array}$ & $\begin{array}{c}\text { AAFRAP } \\
\left(\mathrm{mmol} \mathrm{g}^{-1} \mathrm{DW}\right)\end{array}$ \\
\hline \multicolumn{6}{|l|}{ Accession } \\
\hline CGaz & $0.32^{\mathrm{f}}$ & $2.28^{\mathrm{h}}$ & $0.20^{f}$ & $14.1^{\mathrm{g}}$ & $47.3^{h}$ \\
\hline BaklC & $0.49^{b}$ & $4.76^{\mathrm{a}}$ & $0.41^{\mathrm{a}}$ & $28.8^{\mathrm{a}}$ & $95.4^{\mathrm{a}}$ \\
\hline SisC & $0.32^{\mathrm{f}}$ & $2.57 \mathrm{fg}$ & $0.23^{\mathrm{e}}$ & $14.6^{\mathrm{g}}$ & $50.6^{\mathrm{g}}$ \\
\hline Bel & $0.38^{\mathrm{e}}$ & 2.73 ef & $0.29^{d}$ & $15.9^{f}$ & $56.5^{\mathrm{f}}$ \\
\hline SisS & $0.48^{b c}$ & $2.81^{\mathrm{e}}$ & $0.35^{c}$ & $16.9^{e}$ & $55.6^{f}$ \\
\hline BaklS & $0.48^{b c}$ & $4.46^{\mathrm{b}}$ & $0.41^{\mathrm{a}}$ & $26.8^{\mathrm{b}}$ & $86.0^{\mathrm{c}}$ \\
\hline Kna & $0.38^{\mathrm{e}}$ & $3.26^{\mathrm{d}}$ & $0.29^{\mathrm{d}}$ & $18.6^{\mathrm{c}}$ & $63.9^{\mathrm{e}}$ \\
\hline Bka & $0.31^{\mathrm{f}}$ & $2.45 \mathrm{gh}$ & $0.29 \mathrm{~d}$ & $15.5^{f}$ & $51.7^{g}$ \\
\hline Chba & $0.55^{\mathrm{a}}$ & $4.58^{\mathrm{b}}$ & $0.39 \mathrm{ab}$ & $26.5^{b}$ & $88.1^{\mathrm{b}}$ \\
\hline $\mathrm{FKbK}$ & $0.42^{\mathrm{d}}$ & $3.96^{c}$ & $0.37^{b c}$ & $23.2^{c}$ & $79.3^{d}$ \\
\hline $\mathrm{FKbM}$ & $0.46^{\mathrm{c}}$ & $3.86^{\mathrm{c}}$ & $0.40^{\mathrm{a}}$ & $23.6^{c}$ & $79.8^{\mathrm{d}}$ \\
\hline \multicolumn{6}{|l|}{ Harvest time (DAA) } \\
\hline 10 & $0.26^{f}$ & $2.82^{\mathrm{e}}$ & $0.36^{\mathrm{a}}$ & $16.2^{f}$ & $53.2^{f}$ \\
\hline 25 & $0.59^{b}$ & $2.84^{\mathrm{e}}$ & $0.36^{\mathrm{a}}$ & $16.8^{\text {ef }}$ & $53.8^{f}$ \\
\hline 40 & $0.63^{a}$ & $2.90^{\mathrm{e}}$ & $0.34^{\mathrm{b}}$ & $17.4^{\mathrm{e}}$ & $59.3^{e}$ \\
\hline 55 & $0.34^{\mathrm{d}}$ & $3.07^{\mathrm{d}}$ & $0.34^{\mathrm{b}}$ & $18.7^{\mathrm{d}}$ & $64.7^{\mathrm{d}}$ \\
\hline 70 & $0.29^{e}$ & $3.69^{c}$ & $0.32^{\mathrm{c}}$ & $22.1^{\mathrm{c}}$ & $75.2^{c}$ \\
\hline 85 & $0.26^{\mathrm{f}}$ & $3.87^{b}$ & $0.30^{d}$ & $23.4^{b}$ & $77.6^{\mathrm{b}}$ \\
\hline 100 & $0.45^{\mathrm{c}}$ & $4.80^{\mathrm{a}}$ & $0.28^{\mathrm{e}}$ & $28.3^{a}$ & $96.1^{\mathrm{a}}$ \\
\hline \multicolumn{6}{|l|}{ Source of variation } \\
\hline Accession (A) & $0.15(14.4)^{* * *}$ & $17.90(46.9)^{* * *}$ & $0.12(61.0)^{* * *}$ & $629.79(46.8)^{* * *}$ & $6374.46(43.7)^{* * *}$ \\
\hline Harvest time $(\mathrm{H})$ & $0.82(79.9)^{* * *}$ & $18.12(47.5)^{* * *}$ & $0.03(16.4)^{* * *}$ & $642.56(47.8)^{* * * *}$ & $7907.81(54.2)^{* * *}$ \\
\hline $\mathrm{A} \times \mathrm{H}$ interaction & $0.02(1.6)^{* * *}$ & $0.42(1.1)^{* * *}$ & $0.001(0.6) \mathrm{NS}$ & $12.18(0.9)^{* * *}$ & $190.98(1.3)^{* * *}$ \\
\hline Total mean square & 1.03 & 38.16 & 0.19 & 1344.57 & $14,593.41$ \\
\hline
\end{tabular}

Different letters $\left({ }^{\mathrm{a}-\mathrm{h}}\right)$ within the same trait and main factor indicate significant differences (Duncan's multiple range test, $p<0.05$ ). a For each source of variation (i.e., $\mathrm{A}, \mathrm{H}$ and $\mathrm{A} \times \mathrm{H}$ ) and within each biochemical trait, mean square as absolute value and percentage of total (in brackets) of effects resulting from analysis of variance (ANOVA) were reported. ${ }^{* * *}$ indicates significance at $p<0.001$ and NS indicates non-significance. See caption to Figure 2 for the list of acronyms used for the studied accessions. CAP: capsaicin content. TPC: total phenol content. TFC: total flavonoid content. AADPPH and AAFRAP: antioxidant activity by DPPH and FRAP assays, respectively.

Regarding capsaicin content, our results were not in agreement with those of Silva et al., Gurung et al., Butcher et al., Tripodi et al. or Reddy et al. [35,37-41], reporting a major role of genotype in capsicinoid variation in several Capsicum spp. plants cultivated in different environments across different locations. Our observation for capsaicin content was due probably to the extent of the trial period, allowing environmental fluctuations that influenced the biosynthesis of capsaicin and other compounds. In previous studies by other authors on pepper, nor-dihydrocapsaicin exhibited similar trends, suggesting a role of the environmental conditions in its accumulation [40,42].

\subsection{Chemometrics Analysis}

According to the PCA representations (Figure $4 \mathrm{a}-\mathrm{c}$ ), TPC acted as the greatest contributor to the antioxidant activity at all the harvest times. It was strengthened by the CAP content contribution at 10, 25 and 40 DAA. PC1 was correlated with TP, FRAP and DPPH antioxidant activity. BaklS, BaklC, Chba, FkbM and FkbK were associated with PC1. CAP contributed moderately to the antioxidant activity in comparison with TPC as it was situated far from all the parameters studied. 
(a)

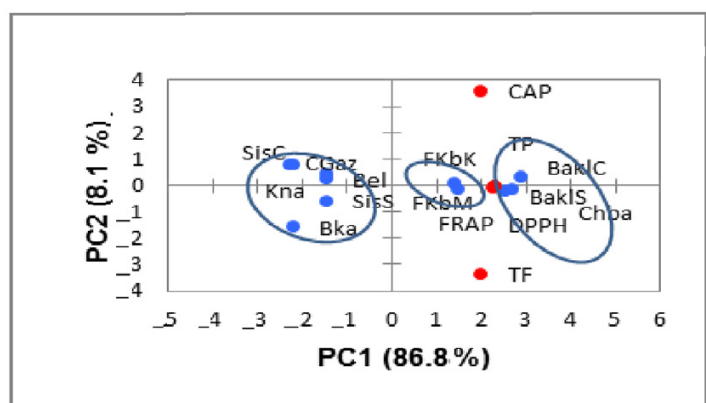

(c)

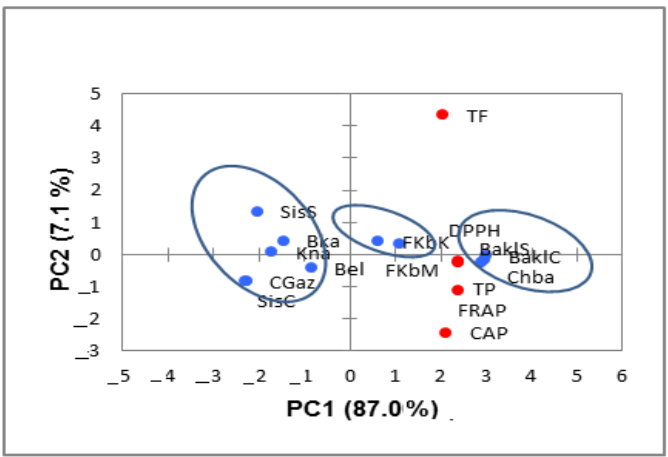

(e)

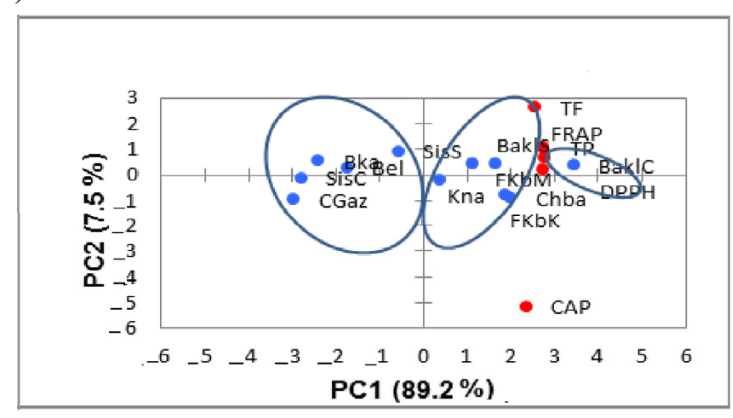

(b)

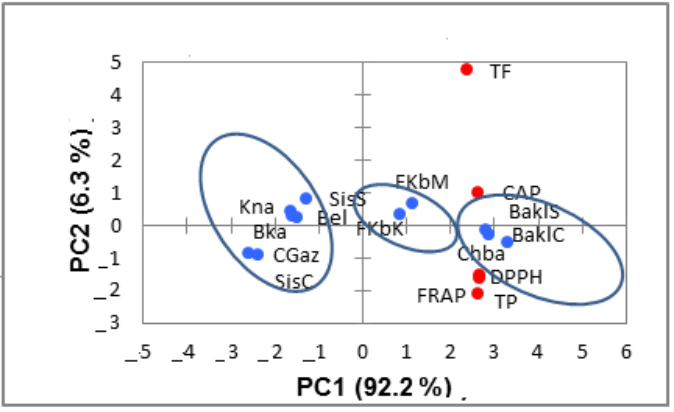

(d)

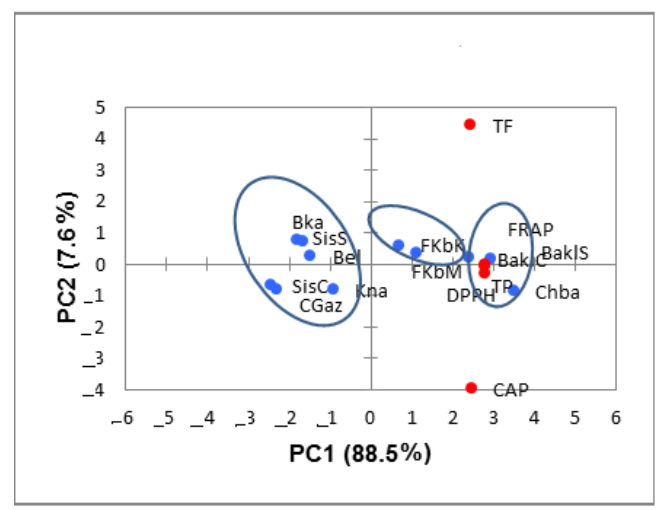

(f)

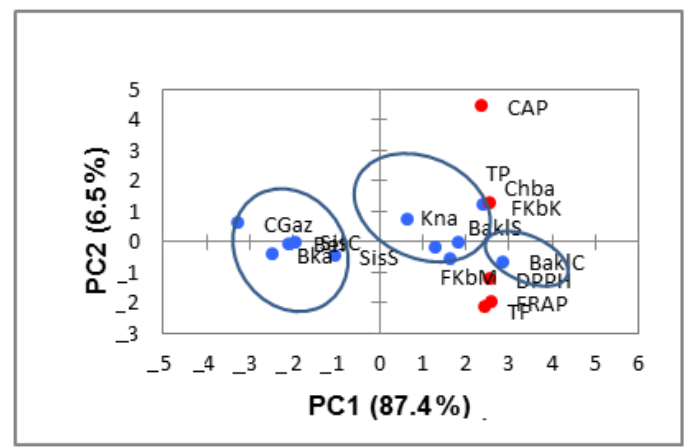

(g)

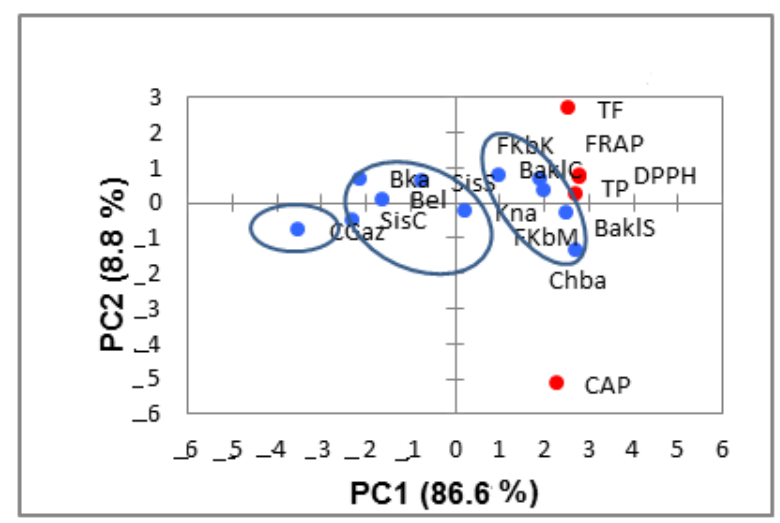

Figure 4. Principal component analysis based on phytochemical compounds and antioxidant activity (by DPPH and FRAP assays) analyzed at 10 (a), 25 (b), 40 (c), 55 (d), 70 (e), 85 (f) and 100 DAA (days after anthesis) (g) in the eleven Tunisian chili pepper accessions. TP: total phenol content. TF: total flavonoid content. CAP: capsaicin content. See caption to Figure 2 for the list of acronyms used for the studied accessions. 
A clear separation of three groups was also observed at 55 (Figure 4d), 70 (Figure 4e), 85 (Figure 4f) and 100 DAA (Figure 4g). PC1 was dominated by AAFRAP, AADPPH, TPC and TFC, which were positively correlated with BaklS, BaklC, Chba and FkbM. In contrast, FkbK and Kna were correlated essentially with CAP content. At 55, 70, 85 and 100 DAA, TPC was the greatest contributor to the total antioxidant activity, with TFC content at a lesser extent, while CAP content seemed to be negligible as it was situated in the extreme part of the biplot (Figure $4 \mathrm{~d}-\mathrm{g}$ ). In addition, at 100 DAA, CGaz formed a separate group, showing the lowest levels of bioactive components and antioxidant activity (Figure $4 \mathrm{~g}$ ).

Based on the seven PCAs (Figure 4) and HCA analysis (Figure 5) representations, CGaz, SisS, SisC, BKa and Bel showed the lowest levels of bioactive components and antioxidant activity, while BaklS, BaklC and Chba presented the highest values. FKbM, FKbK and Kna showed intermediate levels at all the harvest times. TPC and antioxidant activity by both DPPH and FRAP assays were strongly correlated at each harvest time, and this correlation was enhanced as the temperature increased. Indeed, many authors have reported a positive correlation between antioxidant activity and TPC accumulation during temperature exposure, including Kishore et al. and Kumari et al. [43,44]. The type of extraction solvent and the drying method, as well as the antioxidant assays used and the interactions of the antioxidant components, similarly influence the biochemical compound content [45].

\subsection{Correlations}

The correlations among the agronomic and biochemical traits are reported in Table 6. The NF/Pl was negatively correlated with FFW, FL/FD and, particularly, with all the biochemical traits. FFW and NF/Pl were the major traits that directly contributed to yield, as observed by Bozokalfa and Kilic [46]. It also appeared that when pepper plants produced less fruits, the biochemical levels increased, along with the FFW. This was probably related to the source-sink balance in the plant, namely to the competition for assimilates that occurred between fruit yielding and dimensions. Indeed, due to this competition, high flower abortion occurs when fast fruit growth takes place, and fruits from the bottom of the plants are also developed, rather than those from the upper, as observed by Wubs et al. and Zewdie and Bosland [47,48]. Accordingly, plants with a greater fruit size and higher biochemical amounts presented the lowest number of fruits per plant.

(a)

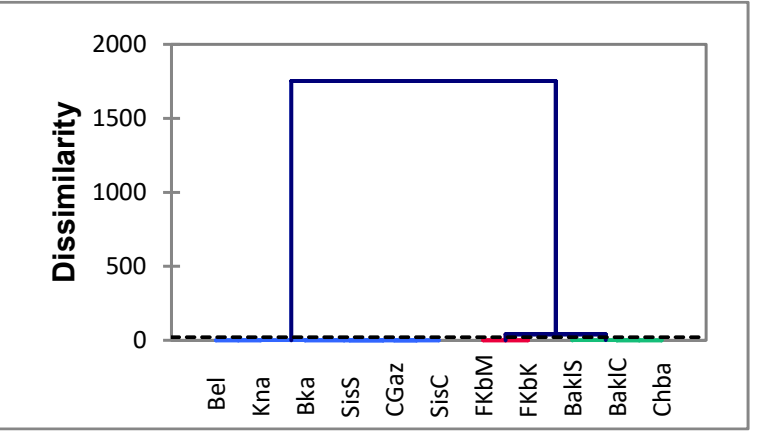

(b)

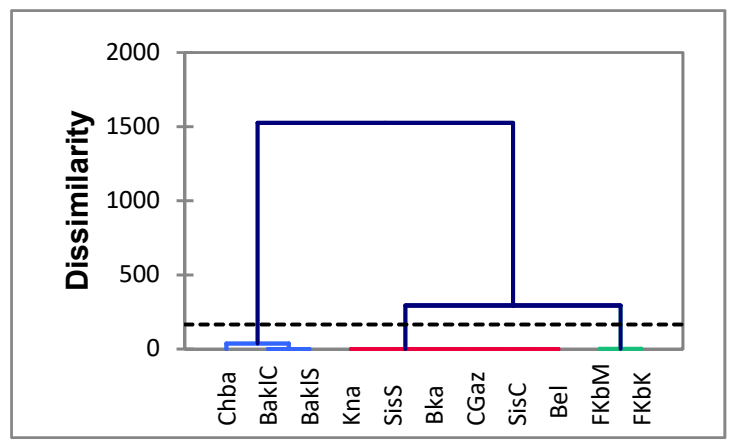

Figure 5. Cont. 
(c)

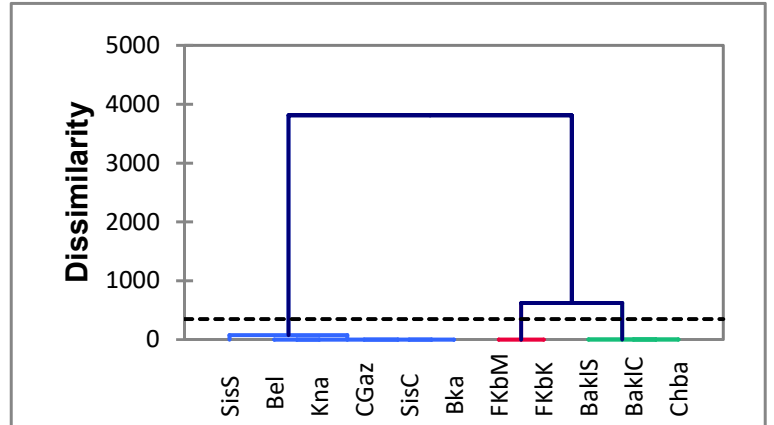

(e)

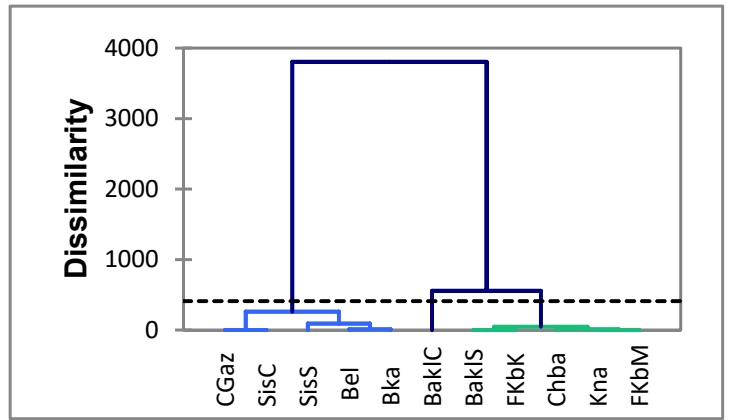

(d)

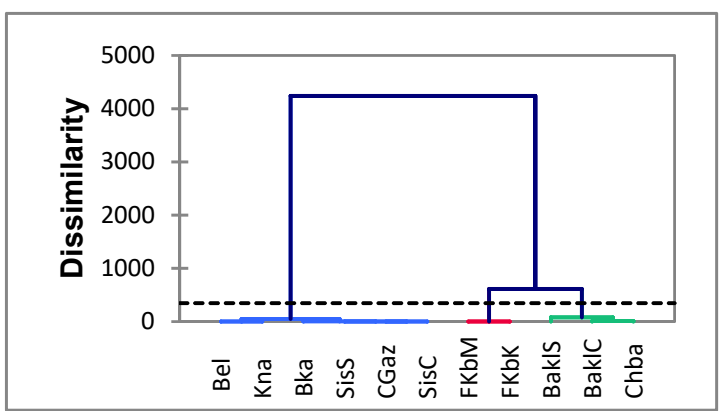

(f)

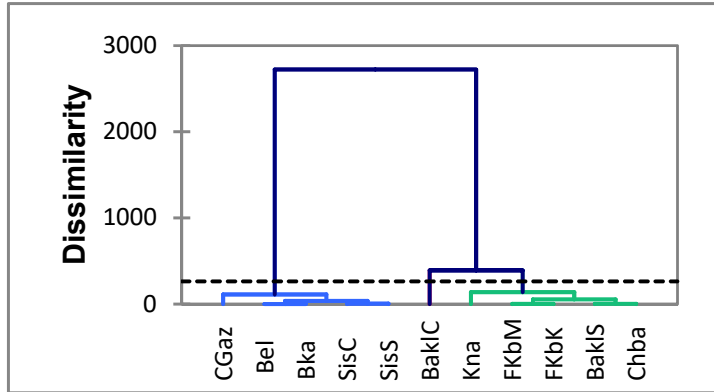

$(\mathrm{g})$

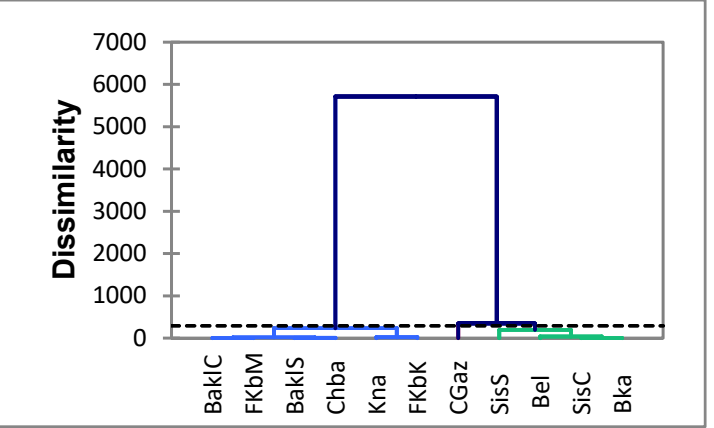

Figure 5. Cluster analysis based on the phytochemical compounds and antioxidant activity (by DPPH and FRAP assays) analyzed at 10 (a), 25 (b), 40 (c), 55 (d), 70 (e), 85 (f) and 100 DAA (days after anthesis) (g) in the eleven Tunisian chili pepper accessions. TPC: total phenol content. TFC: total flavonoid content. CAP: capsaicin content. See caption to Figure 2 for the list of acronyms used for the studied accessions.

Table 6. Bivariate correlations among the studied quantitative traits in the eleven Tunisian pepper accessions.

\begin{tabular}{|c|c|c|c|c|c|c|c|c|}
\hline Variables & FL/FD & FFW & NF/P1 & CAP & ТPC & TFC & AADPPH & AAFRAP \\
\hline $\mathrm{FL} / \mathrm{FD}$ & - & & & & & & & \\
\hline FFW & $0.79^{* * *}$ & - & & & & & & \\
\hline $\mathrm{NF} / \mathrm{Pl}$ & $-0.71^{* *}$ & $-0.87^{* * *}$ & - & & & & & \\
\hline CAP & $-0.09 \mathrm{~ns}$ & $-0.12 \mathrm{~ns}$ & 0.04 ns & - & & & & \\
\hline TPC & $0.24 \mathrm{~ns}$ & $0.25 \mathrm{~ns}$ & -0.42 * & $0.73^{* *}$ & - & & & \\
\hline TFC & $0.13 \mathrm{~ns}$ & $0.26 \mathrm{~ns}$ & -0.32 * & $0.67^{* *}$ & $0.81^{* * *}$ & - & & \\
\hline AADPPH & $0.23 \mathrm{~ns}$ & $0.23 \mathrm{~ns}$ & $-0.42 *$ & $0.73^{* *}$ & $0.95^{* * *}$ & $0.81^{* * *}$ & - & \\
\hline AAFRAP & $0.19 \mathrm{~ns}$ & $0.15 \mathrm{~ns}$ & -0.33 * & $0.80^{* * *}$ & $0.94^{* * *}$ & $0.76^{* *}$ & $0.94^{* * *}$ & - \\
\hline
\end{tabular}

***, ** and * indicate significance at $p \leq 0.001, p \leq 0.01$ and $p \leq 0.05$, and ns indicates non-significance. FL/FD: ratio fruit length (FL, expressed as $\mathrm{cm}$ )/fruit diameter (FD, expressed as $\mathrm{cm}$ ); FFW: fruit fresh weight; NF/Pl: number of fruits per plant; CAP: capsaicin content; TPC: total phenol content; AAFRAP: antioxidant activity (AA) determined by FRAP assays; AADPPH antioxidant activity (AA) determined by DPPH. 
A weak and negative correlation between average fruit width, FFW and CAP content indicated that selection in favor of larger fruit might result in reduced CAP content [49]. Moreover, the accessions tested could provide genes for larger fruits, which is an essential market quality [46]. As expected, considering the phytochemical traits, a high correlation of TPC was detected with antioxidant activity, measured using both the DPPH and FRAP assays. This is in agreement with previous results for other crops [50,51].

\section{Conclusions}

Clear variability existed in terms of plant morphology, with fruit shape and size being the highest contributors to this variation. The high degree of genotypic variability was more pronounced for the biochemical parameters, such as total phenol content and DPPH scavenging activity. Nodal anthocyanin, calyx shape margin, calyx annular constriction and fruit shape at blossom end were discriminative for the tested accessions and were more pronounced within cultivars Kna, Bka, CGaz and SisS. On the other hand, cultivars BaklS, BaklC, Chba, FkbM and FkbK showed high levels of TP, FRAP and DPPH antioxidant activity throughout the harvesting time. PCA and cluster analysis indicated that both agromorphologic and biochemical traits influenced diversity amongst the accessions. Biometric and descriptive traits related to fruit were the most important and valuable traits, useful for further breeding programs. A combination of molecular markers and phenotypic data would be the best choice to further explore the exploitability of local genetic resources and to predict methods to preserve chili pepper germplasm from genetic erosion.

Author Contributions: Data curation, Writing-Original draft preparation, K.L.; Conceptualization, Methodology, Software, S.D.; Software, Validation, F.B.; Writing-Reviewing and Editing, G.P. and S.L.; Supervision, M.E.G.; Resources, Investigation and supervision, S.E.B. All authors have read and agreed to the published version of the manuscript.

Funding: This research received no external funding.

Institutional Review Board Statement: Not applicable.

Informed Consent Statement: Not applicable.

Data Availability Statement: Data are contained within the article.

Acknowledgments: All authors certify that they have no affiliations with or involvement in any organization or entity with any financial interest or non-financial interest in the subject matter or materials discussed in this manuscript.

Conflicts of Interest: The authors declare no conflict of interest. There is no personal circumstances or interest that may be perceived as inappropriately influencing the representation or interpretation of reported research results.

\section{References}

1. Damatta, F.M.; Avila, R.T.; Cardoso, A.A.; Martins, S.C.V.; Ramalho, J.C. Physiological and Agronomic Performance of the Coffee Crop in the Context of Climate Change and Global Warming: A Review. J. Agric. Food Chem. 2018, 66, 5264-5274. [CrossRef]

2. Erickson, A.N.; Markhart, A.H. Flower developmental stage and organ sensitivity of bell pepper (Capsicum annuum L.) to elevated temperature. Plant Cell Environ. 2002, 25, 123-130. [CrossRef]

3. Fan, X.; Cao, X.; Zhou, H.; Hao, L.; Dong, W.; He, C.; Xu, M.; Wu, H.; Wang, L.; Chang, Z.; et al. Carbon dioxide fertilization effect on plant growth under soil water stress associates with changes in stomatal traits, leaf photosynthesis, and foliar nitrogen of bell pepper (Capsicum annuum L.). Environ. Exp. Bot. 2020, 179, 104203. [CrossRef]

4. Rivera, A.; Monteagudo, A.B.; Igartua, E.; Taboada, A.; Garcia-Ulloa, A.; Pomar, F.; Silvar, C. Assessing genetic and phenotypic diversity in pepper (Capsicum annuum L.) landraces from North-West Spain. Sci. Hortic. 2016, 203, 1-11. [CrossRef]

5. Carvalho, S.I.C.; Ragassi, C.F.; Bianchetti, L.B.; Reifschneider, F.J.B.; Buso, G.S.C.; Faleiro, F.G. Morphological and genetic relationships between wild and domesticated forms of peppers (Capsicum frutescens L. and C. chinense Jacquin). Genet. Mol. Res. 2014, 13, 7447-7464. [CrossRef] [PubMed]

6. Chuah, A.M.; Lee, Y.C.; Yamaguchi, T.; Takamura, H.; Yin, L.J.; Matoba, T. Effect of cooking on the antioxidant properties of coloured peppers. Food Chem. 2008, 111, 20-28. [CrossRef]

7. Chandrasekara, A.; Shahidi, F. Determination of antioxidant activity in free and hydrolyzed fractions of millet grains and characterization of their phenolic profiles by HPLC-DAD-ESI-MS. J. Funct. Foods 2011, 3, 144-158. [CrossRef] 
8. $\quad$ Baenas, N.; Belović, M.; Ilic, N.; Moreno, D.A.; García-Viguera, C. Industrial use of pepper (Capsicum annum L.) derived products: Technological benefits and biological advantages. Food Chem. 2019, 274, 872-885. [CrossRef] [PubMed]

9. FAOstat. Food and Agricultural Organisation Statistics. Available online: http://www.fao.org/faostat/en/\#data/QC (accessed on 30 November 2018).

10. Lahbib, K.; Dabbou, S.; Bok, S.E.; Pandino, G.; Lombardo, S.; Gazzah, M.E. Variation of biochemical and antioxidant activity with respect to the part of Capsicum annuum fruit from Tunisian autochthonous cultivars. Ind. Crops Prod. 2017, 104, 164-170. [CrossRef]

11. Bhandari, S.R.; Bashyal, U.; Lee, Y.S. Variations in proximate nutrients, phytochemicals, and antioxidant activity of field-Cultivated red pepper fruits at different harvest times. Hortic. Environ. Biotechnol. 2016, 57, 493-503. [CrossRef]

12. Petropoulos, S.A.; Fernandes, Â.; Antoniadis, V.; Ntatsi, G.; Barros, L.; Ferreira, I.C.F.R. Chemical composition and antioxidant activity of Cichorium spinosum L. leaves in relation to developmental stage. Food Chem. 2018, 239, 946-952. [CrossRef] [PubMed]

13. Lombardo, S.; Pandino, G.; Mauromicale, G. The influence of pre-Harvest factors on the quality of globe artichoke. Sci. Hortic. 2018, 233, 479-490. [CrossRef]

14. IPGRI. Descriptors for Capsicum (Capsicum spp.); International Plant Genetic Resources Institute: Rome, Italy, 1995.

15. Neves, L.C.; de Campos, A.J.; Colombo, R.C.; Roberto, S.R.; Cisneros-Zevallos, L. Days after anthesis and postharvest behavior define maturity, harvesting time and nutraceutical content of camu-camu fruit. Sci. Hortic. 2017, 224, 37-47. [CrossRef]

16. Sadasivam, S.; Manikkam, A. Capsaicin. Biochem. Methods Agric. Sci. 1992, 8, 193-194.

17. Singleton, V.L.; Rossi, J.A.J. Colorimetry of total phenolics with phosphomolybdic-Phosphotungstic acid reagents. Am. J. Enol. Vitic. 1965, 16, 144-158. [CrossRef]

18. Um, H.-J.; Kim, G.-H. Studies on the flavonoid compositions of Elsholtzia spp. Korean J. Food Nutr. 2007, 20, $103-107$.

19. Brand-Williams, W.; Cuvelier, M.E.; Berset, C. Use of a free radical method to evaluate antioxidant activity. LWT-Food Sci. Technol. 1995, 28, 25-30. [CrossRef]

20. Benzie, I.F.F.; Strain, J.J. The ferric reducing ability of plasma (FRAP) as a Measure of "Antioxidant Power": The FRAP assay. Anal. Biochem. 1996, 76, 70-76. [CrossRef]

21. Mazzucato, A.; Papa, R.; Bitocchi, E.; Mosconi, P.; Nanni, L.; Negri, V.; Veronesi, F. Genetic diversity, structure and marker-trait associations in a collection of Italian tomato (Solanum lycopersicum L.) landraces. Theor. Appl. Genet. 2008, 116, 657-669. [CrossRef] [PubMed]

22. Mercati, F.; Longo, C.; Poma, D.; Araniti, F.; Lupini, A.; Mammano, M.M.; Sunseri, F. Genetic variation of an Italian long shelf-Life tomato (Solanum lycopersicon L.) collection by using SSR and morphological fruit traits. Genet. Resour. Crop Evol. 2014, 62, 721-732. [CrossRef]

23. Yumnam, J.S.; Tyagi, W.; Pandey, A.; Meetei, N.T.; Rai, M. Evaluation of Genetic Diversity of Chilli Landraces from North Eastern India Based on Morphology, SSR Markers and the Pun1 Locus. Plant Mol. Biol. Report. 2012, 30, 1470-1479. [CrossRef]

24. Bozokalfa, M.K.; Esiyok, D.; Turhan, K. Patterns of phenotypic variation in a germplasm collection of pepper (Capsicum annuum L.) from Turkey. Span. J. Agric. Res. 2009, 7, 83-95. [CrossRef]

25. Anjum, S.A.; Farooq, M.; Xie, X.Y.; Liu, X.J.; Ijaz, M.F. Antioxidant defense system and proline accumulation enables hot pepper to perform better under drought. Sci. Hortic. 2012, 140, 66-73. [CrossRef]

26. Fayos, O.; De Aguiar, A.C.; Jiménez-Cantizano, A.; Ferreiro-González, M.; Garcés-Claver, A.; Martínez, J.; Mallor, C.; Rodríguez, A.R.; Palma, M.; Barroso, C.G.; et al. Ontogenetic variation of individual and total capsaicinoids in Malagueta peppers (Capsicum frutescens) during fruit maturation. Molecules 2017, 22, 736. [CrossRef]

27. Barbero, G.F.; Ruiz, A.G.; Liazid, A.; Palma, M.; Vera, J.C.; Barroso, C.G. Evolution of total and individual capsaicinoids in peppers during ripening of the Cayenne pepper plant (Capsicum annuum L.). Food Chem. 2014, 153, 200-206. [CrossRef] [PubMed]

28. Ruiz-Lau, N.; Medina-Lara, F.; Minero-García, Y.; Zamudio-Moreno, E.; Guzmán-Antonio, A.; Echevarría-Machado, I.; MartínezEstévez, M. Water Deficit Affects the Accumulation of Capsaicinoids in Fruits of Capsicum chinense Jacq. Hortic. Sci. 2011, 46, 487-492. [CrossRef]

29. Que, F.; Mao, L.; Fang, X.; Wu, T. Comparison of hot air-Drying and freeze-Drying on the physicochemical properties and antioxidant activities of pumpkin (Cucurbita moschata Duch.) flours. Int. J. Food Sci. Technol. 2008, 43, 1195-1201. [CrossRef]

30. Ghasemnezhad, M.; Sherafati, M.; Payvast, G.A. Variation in phenolic compounds, ascorbic acid and antioxidant activity of five coloured bell pepper (Capsicum annum) fruits at two different harvest times. J. Funct. Foods 2011, 3, 44-49. [CrossRef]

31. Howard, L.R.; Talcott, S.T.; Brenes, C.H.; Villalon, B. Changes in phytochemical and antioxidant activity of selected pepper cultivars (Capsicum species) as influenced by maturity. J. Agric. Food Chem. 2000, 48, 1713-1720. [CrossRef] [PubMed]

32. Steyn, W.J.; Wand, S.J.E.; Jacobs, G.; Rosecrance, R.C.; Roberts, S.C. Evidence for a photoprotective function of low-Temperatureinduced anthocyanin accumulation in apple and pear peel. Physiol. Plant. 2009, 136, 461-472. [CrossRef] [PubMed]

33. Pandino, G.; Lombardo, S.; Lo Monaco, A.; Mauromicale, G. Choice of time of harvest influences the polyphenol profile of globe artichoke. J. Funct. Foods 2013, 5, 1822-1828. [CrossRef]

34. Mitic, V.D.; Cvetkovic, J.S.; Stankov-Jovanovic, V.P.; Dimitrijevic, M.V.; Stojanovic, G.S. Characterization of Pepper Genotypes from Serbia as a Function of Maturity by Antioxidant Activity with Chemometric Analysis. Anal. Lett. 2016, 49, $2234-2245$. [CrossRef] 
35. Silva, R.; Silva, C.; Francisco, C.; Isabel, S.; Carvalho, C.; De Rodrigues, I.; Da Silva Filho, J.G.; Trevisan Braz, L.; Beckercd, F.J.; Reifschneider, B. New Brazilian lines of Habanero pepper Capsicum chinense: Morpho-Agronomic and biochemical characterization in different environments. Sci. Hortic. 2019, 261, 108941. [CrossRef]

36. Meckelmann, S.W.; Riegel, D.W.; van Zonneveld, M.; Ríos, L.; Peña, K.; Mueller-Seitz, E.; Petz, M. Capsaicinoids, flavonoids, tocopherols, antioxidant capacity and color attributes in 23 native Peruvian chili peppers (Capsicum spp.) grown in three different locations. Eur. Food Res. Technol. 2014, 240, 273-283. [CrossRef]

37. Gurung, T.; Techawongstien, S.; Suriharn, B.; Techawongstien, S. Impact of environments on the accumulation of capsaicinoids in Capsicum spp. Hortic. Sci. 2011, 46, 1576-1581. [CrossRef]

38. Butcher, J.D.; Crosby, K.M.; Yoo, K.S.; Patil, B.S.; Leskovar, D.I.; Jifon, J.L. Environmental and Genotypic Variation of Capsaicinoid and Flavonoid Concentrations in Habanero (Capsicum chinense) Peppers. HortScience 2012, 47, 574-579. [CrossRef]

39. Gurung, T.; Techawongstien, S.; Suriharn, B.; Techawongstien, S. Stability analysis of yield and capsaicinoids content in chili (Capsicum spp.) grown across six environments. Euphytica 2012, 187, 11-18. [CrossRef]

40. Tripodi, P.; Cardi, T.; Bianchi, G.; Migliori, C.A.; Schiavi, M.; Rotino, G.L.; Lo Scalzo, R. Genetic and environmental factors underlying variation in yield performance and bioactive compound content of hot pepper varieties (Capsicum annuum) cultivated in two contrasting Italian locations. Eur. Food Res. Technol. 2018, 244, 1555-1567. [CrossRef]

41. Reddy, U.K.; Almeida, A.; Abburi, V.L.; Alaparthi, S.B.; Unselt, D.; Hankins, G.; Park, M.; Choi, D.; Nimmakayala, P. Identification of Gene-Specific Polymorphisms and Association with Capsaicin Pathway Metabolites in Capsicum annuum L. Collections. PLoS ONE 2014, 9, e86393. [CrossRef]

42. Zewdie, Y.; Bosland, P.W. Evaluation of genotype, environment, and genotype-By-Environment interaction for capsaicinoids in Capsicum annuum L. Euphytica 2000, 111, 185-190. [CrossRef]

43. Kishore, G.; Ranjan, S.; Pandey, A.; Gupta, S. Influence of altitudinal variation on the antioxidant potential of tartar buckwheat of Western Himalaya. Food Sci. Biotechnol. 2010, 19, 1355-1363. [CrossRef]

44. Kumari, D.; Madhujith, T.; Chandrasekara, A. Comparison of phenolic content and antioxidant activities of millet varieties grown in different locations in Sri Lanka. Food Sci. Nutr. 2016, 5, 474-485. [CrossRef]

45. Chen, M.L.; Yang, D.J.; Liu, S.C. Effects of drying temperature on the flavonoid, phenolic acid and antioxidative capacities of the methanol extract of citrus fruit (Citrus sinensis (L.) Osbeck) peels. Int. J. Food Sci. Technol. 2011, 46, 1179-1185. [CrossRef]

46. Bozokalfa, M.K.; Kilic, M. Mathematic medeling in the estimation of pepper (Capsicum annuum L.) fruit volume. Chil. J. Agric. Res. 2010, 70, 626-632. [CrossRef]

47. Wubs, A.M.; Heuvelink, E.; Marcelis, L.F.M. Abortion of reproductive organs in sweet pepper (Capsicum annuum L.): A review. J. Hortic. Sci. Biotechnol. 2016, 84, 467-475. [CrossRef]

48. Zewdie, Y.; Bosland, P.W. Pungency of Chile (Capsicum annuum L.) Fruit Is Affected by Node Position. Hortic. Sci. 2000, $35,1174$. [CrossRef]

49. Anyaoha, C.O.; Ademoyegun, O.T.; Solomon, O.; Anyaoha, C.O. Agro-Morphological and nutritional variability among elite Nsukka yellow spice pepper breeding lines Agro-Morphological and nutritional variability among elite. Int. J. Veg. Sci. 2019, 516-531. [CrossRef]

50. Pandino, G.; Meneghini, M.; Tavazza, R.; Lombardo, S.; Mauromicale, G. Phytochemicals accumulation and antioxidant activity in callus and suspension cultures of Cynara scolymus L. Plant Cell Tissue Organ. Cult. 2017, 128, 223-230. [CrossRef]

51. Dabbou, S.; Lahbib, K.; Pandino, G.; Dabbou, S.; Lombardo, S. Evaluation of pigments, phenolic and volatile compounds, and antioxidant activity of a spontaneous population of Portulaca oleracea L. grown in Tunisia. Agriculture 2020, 10, 353. [CrossRef] 